\title{
MATRIX TRANSFORMATIONS OF UNIVALENT POWER SERIES
}

\author{
F. W. HARTMANN and T. H. MACGREGOR 1
}

(Received 14 February 1973)

Communicated by E. Strzelecki

\section{Introduction}

Suppose that $A=\left[\alpha_{n k}\right],(n, k=0,1,2, \cdots)$, is an infinite matrix with complex entries. $A$ transforms a complex sequence $a=\left\{a_{n}\right\}$ to a complex sequence $\left\{b_{n}\right\}=b=A a$ where

$$
b_{n}=\sum_{k=0}^{\infty} \alpha_{n k} a_{k} \quad n=0,1,2, \cdots
$$

assuming that the series in (1) converges. Each sequence $a=\left\{a_{n}\right\}$ is uniquely associated with a power series

$$
f(z)=\sum_{n=0}^{\infty} a_{n} z^{n}
$$

In this way the matrix $A$ transforms a power series into a power series. Specifically, the power series (2) is mapped to the power series

$$
g(z)=\sum_{n=0}^{\infty} b_{n} z^{n}
$$

where the $b_{n}$ 's are given by (1). We are only interested in matrices having the property that each power series analytic in $\Delta=\{z:|z|<1\}$ maps to a power series analytic in $\Delta$.

Our concern is motivated by the general question: What matrices map a function $f$ univalent in $\Delta$ to another function $g$ univalent in $\Delta$ ? We shall consider three specific, well-known matrices and study to what extent properties of univalence of $f$ are carried over to $g$. These matrices are:

1 The research of the second author was partially supported by a National Science Foundation Grant and a State University of New York Faculty Fellowship. 
(4)

$$
\begin{aligned}
& (C, 1)=\left[\alpha_{n k}\right] ; \quad \alpha_{O k}=0, k=0,1,2, \cdots \\
& \alpha_{n 0}=0, n=1,2, \cdots \\
& \alpha_{n k}= \begin{cases}\frac{1}{n}, & \text { if } k \leqq n, n=1,2, \cdots \\
0, & \text { if } k>n, n=1,2, \cdots\end{cases} \\
& E(r)=\left[\alpha_{n k}\right], \quad 0<r<1, \\
& \alpha_{n k}= \begin{cases}\left(\begin{array}{l}
n \\
k
\end{array}\right) r^{k}(1-r)^{n-k}, & \text { if } k \leqq n \\
0, & \text { if } k>n\end{cases} \\
& T(r)=\left[\alpha_{n k}\right], \quad 0<r<1, \\
& \alpha_{n k}= \begin{cases}0, & \text { if } n>k \\
(1-r)^{n+1}\left(\begin{array}{l}
k \\
n
\end{array}\right) r^{k-n}, & \text { if } k \geqq n .\end{cases}
\end{aligned}
$$

The $(\mathrm{C}, 1)$ matrix shall be called the Cesàro transformation $[6$, page 175$] ; E(r)$ the Euler-Knopp transformation [6, page 176] and $T(r)$ the Taylor transformation [1]. Equation (1) becomes

$$
\begin{array}{ll}
b_{n}=\frac{1}{n} \sum_{k=1}^{n} a_{k} & (n=1,2, \cdots), b_{0}=0 \\
b_{n}=\sum_{k=0}^{\infty}\left(\begin{array}{l}
n \\
k
\end{array}\right) r^{k}(1-r)^{n-k} a_{k} & (n=0,1,2, \cdots)
\end{array}
$$

and

$$
b_{n}=\sum_{k=n}^{\infty}(1-r)^{n+1}\left(\begin{array}{l}
k \\
n
\end{array}\right) r^{k-n} a_{k} \quad(n=0,1,2, \cdots)
$$

for the matrices (4), (5) and (6), respectively. One can show that each of these matrices maps a function analytic in $\Delta$ to a function analytic in $\Delta$. This fact is a simple consequence of Hadamard's formula for the radius of convergence of a power series.

Each of the transformations, $(C, 1), E(r), T(r)$, preserves various properties of univalence. Specifically, if $f$ is univalent in $\Delta$ and $f(\Delta)$ is convex then the Cesàro transform $g$ also maps $\Delta$ one-to-one onto a convex domain. If $f$ maps $\Delta$ one-to-one onto a domain that is starlike with respect to the origin, then the Cesàro transform of $f$ is univalent in $\Delta$. We also give a number of examples of functions $f$ univalent in $\Delta$ so that $g$ is univalent in $\Delta$ and finally an example of a univalent function whose Cesàro transform is not univalent.

Similar results are obtained for the Euler-Knopp transform. We find that if $f$ 
is univalent and starlike in $\Delta$ then so is the transform $g$. We also show that if $g$ is suitably normalized then it satisfies a number of distortion theorems and coefficient inequalities for univalent functions. Our study of $E(r)$ concludes with an example of a univalent function whose $E(r)$ transform is not univalent.

On a positive note, however, we show that if $f$ is univalent, its Taylor transform $g$ is always univalent and moreover $g$ satisfies many of the coefficient inequalities known to hold for the suitably normalized univalent functions.

\section{The Cesàro transformation}

In this section we consider what properties of univalence are preserved in the transformation from the functions (2) to the functions (3) where $b_{n}$ is given by (7). We begin by observing a simple relation between $f$ and $g$. Namely,

$$
\begin{aligned}
g^{\prime}(z) & =\sum_{n=1}^{\infty} n b_{n} z^{n-1}=\sum_{n=1}^{\infty}\left\{\sum_{k=1}^{n} a_{k}\right\} z^{n-1} \\
& =\left\{\sum_{k=0}^{\infty} z^{k}\right\}\left\{\sum_{n=1}^{\infty} a_{n} z^{n-1}\right\}=\frac{1}{1-z} \frac{f(z)}{z} . \quad(z \neq 0) .
\end{aligned}
$$

Therefore,

$$
g^{\prime}(z)=\frac{1}{1-z} \frac{f(z)}{z},(z \neq 0), \quad\left(g^{\prime}(0)=f^{\prime}(0)\right)
$$

or, equivalently, given that $g(0)=0$,

$$
g(z)=\int_{0}^{z} \frac{1}{1-w} \frac{f(w)}{w} d w .
$$

THEOREM 1. If $f$ is analytic in $\Delta$ and maps $\Delta$ one-to-one onto a convex domain, then the Cesàro transform $g$ of $f$ is also analytic, univalent and convex in $\Delta$.

Proof. A function $f$ analytic in $\Delta$ and satisfying $f^{\prime}(0) \neq 0$ is univalent and convex if and only if

$$
\operatorname{Re}\left\{\frac{z f^{\prime \prime}(z)}{f^{\prime}(z)}+1\right\}>0 \text { for }|z|<1 .
$$

Such functions are known to be starlike of order $\frac{1}{2}$ as proved in [7] and [11]; that is,

$$
\operatorname{Re}\left\{\frac{z f^{\prime}(z)}{f(z)}\right\}>\frac{1}{2} \text { for }|z|<1 \text {. }
$$

Now, let $f$ be analytic, univalent and convex in $\Delta$ and let $g$ be the Cesàro transform of $f$. Then $f$ and $g$ are related through equation (10) from which we 
find that

$$
\frac{z g^{\prime \prime}(z)}{g^{\prime}(z)}+1=\frac{z f^{\prime}(z)}{f(z)}+\frac{z}{1-z} .
$$

Since $f$ satisfies equation (13) and $w=z(1-z)$ maps $\Delta$ (one-to-one) onto $\operatorname{Re} w>$ $-\frac{1}{2}$ we conclude from (14) that

$$
\operatorname{Re}\left\{\frac{z g^{\prime \prime}(z)}{g^{\prime}(z)}+1\right\}>\frac{1}{2}-\frac{1}{2}=0 \text { for }|z|<1 .
$$

As $g^{\prime}(0)=f^{\prime}(0) \neq 0$ this proves that $g$ maps $\Delta$ one-to-one onto a convex domain.

Our argument shows more than the assertion of Theorem 1; namely, if $f$ is starlike of order $\frac{1}{2}$ then $g$ is univalent and convex. More generally, if $f$ is starlike of order $\alpha$ then $g$ is convex of order $\alpha-\frac{1}{2}$; that is, the inequality

$$
\operatorname{Re}\left\{\frac{z f^{\prime}(z)}{f(z)}\right\}>\alpha \text { for }|z|<1
$$

implies that

$$
\operatorname{Re}\left\{\frac{z g^{\prime \prime}(z)}{g^{\prime}(z)}+1\right\}>\beta \text { for }|z|<1
$$

with $\beta=\alpha-\frac{1}{2}$. It is known that the condition (16) with $\beta \geqq-\frac{1}{2}$ (and $g^{\prime}(0) \neq 0$ ) implies that $g$ is univalent in $\Delta[12]$. Thus, if $f$ is starlike of order zero (or, equivalently, $f$ is univalent in $\Delta$ and $f(\Delta)$ is starlike with respect to the origin) then $g$, the Cesàro transform of $f$, is univalent in $\Delta$. We now state this result and give an alternative proof of it.

THEOREM 2. If $f$ is analytic in $\Delta, f(0)=0$, and if $f$ maps $\Delta$ one-to-one onto a starlike domain, then the Cesàro transformation of $f$ is also univalent in $\Delta$.

Proof. Let $g$ be the Cesàro transformation of $f$. Then, equation (10) holds and since $w=1 /(1-z)$ maps (one-to-one) onto $\operatorname{Re} w>\frac{1}{2}$ this shows that

$$
\operatorname{Re}\left\{\frac{z g^{\prime}(z)}{f(z)}\right\}>\frac{1}{2} \text { for }|z|<1 .
$$

In particular, this implies that

$$
\operatorname{Re}\left\{\frac{z g^{\prime}(z)}{f(z)}\right\}>0 \text { for }|z|<1 .
$$

Equation (18) is the definition that $g$ be close-to-convex in $\Delta$, since $f$ is univalent and starlike and $f(0)=0$. Each close-to-convex function is univalent in $\Delta$ as proved by Kaplan in [3].

Theorem 2 provides us with numerous examples of univalent functions 
whose Cesàro transformation is also univalent since starlike univalent functions can be easily constructed through the condition $\operatorname{Re}\left\{z f^{\prime}(z) / f(z)\right\}>0$. For example, $f(z)=z /(1-z)^{\alpha}$ is univalent and starlike in $\Delta$ if $0 \leqq \alpha \leqq 2$ and the Cesàro transformations are

$$
g(z)=\frac{1}{\alpha}\left\{(1-z)^{-\alpha}-1\right\} .
$$

Other examples are given by the following assertion which is generally known and which we need to use in our discussion of the Euler-Knopp transformation.

Lemma 1. Suppose that $\lambda_{k}$ are real numbers and $\varepsilon_{k}$ are complex numbers for $k=1,2, \cdots, n$ so that

Then

$$
\lambda_{k} \geqq 0, \quad \sum_{k=1}^{n} \lambda_{k} \leqq 2 \text { and }\left|\varepsilon_{k}\right| \leqq 1 .
$$

$$
f(z)=\frac{z}{\prod_{k=1}^{n}\left(1-\varepsilon_{k} z\right)^{\lambda_{k}}}
$$

is univalent and starlike in $\Delta$.

We also consider the examples

$$
f(z)=\frac{z-a z^{2}}{(1-z)^{2}}
$$

which shall later be of importance in obtaining a counter-example with regard to the Euler-Knopp transformation. The function in equation (19) is univalent in $\Delta$ if and only if $a$ satisfies the inequality

$$
\left|a-\frac{1}{2}\right| \leqq \frac{1}{2}
$$

This fact is generally known but we indicate a simple proof of it here. That (20) is necessary for the univalence of $f$ follows from the fact that $f^{\prime}(z)$ vanishes at $z=1 /(2 a-1)$ and this number is in $\Delta$ if $(20)$ is not satisfied. Since the derivative of a univalent function cannot vanish, this proves the necessity of (20). Conversely, suppose that (20) holds. If $b \neq 0$ then the function $F(z)=b z /(1-z)^{2}$ is univalent and starlike in $\Delta$, and

$$
\frac{z f^{\prime}(z)}{F(z)}=\frac{1}{b} \frac{1+(1-2 a) z}{1-z} .
$$

The function $w=[1+(1-2 a) z] /(1-z)$ maps $\Delta$ onto some half-plane and by the hypothesis (20) that half-plane does not contain the origin. Thus, for some 
complex number $b$ the right hand side of equation (21) satisfies $\operatorname{Re} w>0$ for $z$ in $\Delta$. This shows that $\operatorname{Re}\left\{z f^{\prime}(z) / F(z)\right\}>0$ in $\Delta$. Thus, $f$ is close-to-convex and, hence, univalent in $\Delta$.

Let $f$ be defined by equation (19) and let inequality (20) hold so that $f$ is univalent in $\Delta$. If $g$ is the Cesàro transformation of $f$, then from equation (11) and some integration we find that

$$
g(z)=\frac{z-\frac{a+1}{2} z^{2}}{(1-z)^{2}}
$$

Thus $g$ has the same form as $f$ where $a$ has been replaced by $b=\frac{1}{2}(a+1)$. Inequality (20) is equivalent to $\left|b-\frac{3}{4}\right| \leqq \frac{1}{4}$ and so, in particular, $\left|b-\frac{1}{2}\right| \leqq \frac{1}{2}$. Therefore, $g$ is also univalent in $\Delta$.

Although the Cesàro transformation of a convex, univalent mapping is also convex it is not the case that the Cesàro transformation preserves starlike mappings. We give a simple example to show this. Namely, the function $f(z)=z /(1-z)^{2}$ is univalent and starlike in $\Delta$ and, according to equation (22) with $a=0$, the Cesàro transformation of this function is

$$
g(z)=\frac{z-\frac{1}{2} z^{2}}{(1-z)^{2}} .
$$

From (23) we find that

$$
\frac{z g^{\prime}(z)}{g(z)}=\frac{1-z}{1-\frac{1}{2} z}+\frac{2 z}{1-z} .
$$

If $z \neq 1$ and $|z|=1$ then $\operatorname{Re} 2 z /(1-z)=-1$. Thus, as $w=(1-z) /\left(1-\frac{1}{2} z\right)$ vanishes at $z=1$, there are numbers $z$ sufficiently close to 1 so that $|z|=1$ and $\operatorname{Re}\left\{z g^{\prime}(z) / g(z)\right\}$ is as near to -1 as we like. Accordingly, there are points in $\Delta$ so that $\operatorname{Re}\left\{z g^{\prime}(z) / g(z)\right\}<0$ and, therefore, $g$ is not starlike (although univalent) in $\Delta$.

Our final result about the Cesàro transformation is an example of a univalent function whose transformation is not univalent. We need recall that if $f$ is analytic in $\Delta, f(0)=0, f^{\prime}(0) \neq 0$ and

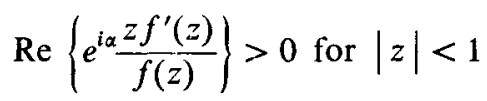

where $\alpha$ is real $(|\alpha|<\pi / 2)$, then $f$ is univalent in $\Delta[$ see 10$]$. These conditions are satisfied by the function

$$
f(z)=z \exp \{-a \log (1+z)\}
$$

where $a=2 e^{-i \alpha} \cos \alpha$. In particular, note that (26) implies that 


$$
\operatorname{Re}\left\{e^{i \alpha} \frac{z f^{\prime}(z)}{f(z)}\right\}=\operatorname{Re}\left\{\frac{e^{i \alpha}-e^{-i \alpha} z}{1+z}\right\}=\cos \alpha \operatorname{Re}\left\{\frac{1-z}{1+z}\right\}>0 .
$$

Our example is the function (26) for any choice of $\alpha(\alpha \neq 0)$. This kind of function has served as a counter-example for some earlier questions [see 4 and 5].

THEOREM 3. There is a function $f$ that is analytic and univalent in $\Delta$ so that $f(0)=0$ and the Cesàro transformation of $f$ is not univalent in $\Delta$.

Proof. Let $f$ be given by (26) where $a=2 e^{-i \alpha} \cos \alpha,|\alpha|<\pi / 2, \alpha \neq 0$ and let $g$ be the Cesàro transformation of $f$. Then, by (11) we may write

$$
g(z)=\int_{0}^{z} \frac{1}{(1-w)(1+w)^{a}} d w .
$$

We shall show that $g$ is not univalent by studying its local behavior at $z=1$ and $z=-1$ for points in $\Delta$.

The derivative of $g$ is analytic for $|z| \leqq 1$ except at the points $z=1$ and $z=-1$ and at $z=1$ it has a simple pole. We may write $g^{\prime}(z)=A /(1-z)+h(z)$ for some constant $A$ and some function $h$ analytic on some neighborhood of the segment $[0,1]$. By integrating along the line segment from 0 to $z$ for each $z$ in this neighborhood we conclude that $g(z)=-A \log (1-z)+k(z)$, where $z$ is not on the real axis from 1 to $\infty$ and $k$ is analytic at $z=1$. The image of the segment $[0,1)$ under the mapping $w=-A \log (1-z)$ is a ray. Thus, the image of this segment under $g$ is an analytic curve $C$ asymptotic to some ray.

Next we study $g$ at $z=-1$. If the integral in (27) is rewritten through two integrations by parts we conclude that

$$
\begin{aligned}
g(z)= & \frac{(1+z)^{-a+1}}{(-a+1)(1-z)}-\frac{1}{-a+1}-\frac{(1+z)^{-a+2}}{(-a+1)(-a+2)(1-z)^{2}} \\
& +\frac{1}{(-a+1)(-a+2)}+\frac{2}{(-a+1)(-a+2)} \int_{0}^{z} \frac{(1+w)^{-a+2}}{(1-w)^{3}} d w .
\end{aligned}
$$

The last term in this equation may be written

$$
Q(z)=\int_{0}^{z}(1+w)^{-a+2} P(w) d w
$$

where $P$ is analytic for $|z| \leqq 1$ except at $z=1$. Suppose that

$$
(1+z)^{-a+2}=\sum_{n=0}^{\infty} C_{n} z^{n} \text { for }|z|<1
$$

so that $C_{n}=\left(\begin{array}{c}-a+2 \\ n\end{array}\right)$. If $b$ is any complex number other than a non-positive integer then $n ! n^{b} /[b(b+1)(b+2) \cdots(b+n)] \rightarrow \Gamma(b)$ as $n \rightarrow \infty$, and thus 


$$
\left|C_{n} / n^{a-3}\right| \rightarrow|\Gamma(a-2)|^{-1} \text { as } n \rightarrow \infty \text {. }
$$

Since $\left|n^{a-3}\right|=n^{R e(a-3)}=n^{2 \cos ^{2} \alpha-3}$ and $\alpha \neq 0,|\alpha|<\pi / 2$, we conclude that $\left|C_{n}\right|=0\left(n^{-\beta}\right)$ for some $\beta>1$. Thus, the power series in (30) converges absolutely for $|z| \leqq 1$, and thereby the function $w=(1+z)^{-a+2}$ extends continuously for $|z| \leqq 1$. Equation (29) then implies that $Q$ is continuous for $|z| \leqq 1$ except at $z=1$, and so, in particular, $Q$ is continuous at $z=-1$ with respect to points in $\Delta$. Therefore, by combining various terms we may rewrite equation (28) into the form

$$
g(z)=(1+z)^{-a+1} R(z)+S(z)
$$

where $R$ and $S$ are continuous at $z=-1$ and $R(-1) \neq 0$.

The image of the interval $(-1,0)$ under the mapping $w=\log (1+z)$ is the interval $(-\infty, 0)$. By the mapping $\zeta=(-a+1) w=-e^{-2 i \alpha_{w}}$ the interval $(-\infty, 0)$ transforms into a ray emanating from $\zeta=0$ which lies in the right-half plane $\operatorname{Re} \zeta>0$ and is not the positive real axis, because $|\alpha|<\pi / 2$ and $\alpha \neq 0$. That ray is mapped onto a spiral from $s=0$ and spiralling to $s=\infty$ under the function $s=e^{\zeta}$. This shows that $s=(1+z)^{-a+1}$ maps the interval $(-1,0)$ onto such a spiral. Because of equation (31) we conclude that $g$ maps $(-1,0)$ onto a curve $C^{\prime}$ that is asymptotic to a spiral beginning at some finite point and spiralling to $\infty$.

The properties of the curves $C$ and $C^{\prime}$ imply that they must intersect (in fact, infinitely often). That is, there is a complex number $w$ such that $w=g\left(z_{1}\right)$ and $w=g\left(z_{2}\right)$ where $z_{1} \in[0,1)$ and $z_{2} \in(-1,0)$. Therefore, $g$ is not univalent in $\Delta$.

It is interesting to note that if we let $f(z)=z \exp \{-a \log (1-z)\}$ where $a=2 e^{-i \alpha} \cos \alpha(|\alpha|<\pi / 2)$ then condition (25) holds and so $f$ is univalent in $\Delta$. For this spiral-like function we find that its Cesàro transformation is univalent in $\Delta$.

\section{The Euler-Knopp transformation}

We now study questions of the preservation of properties of univalence for the transformation of power series given by the Euler-Knopp matrix (5). This transforms the power series (2) into (3) where equation (8) relates the coefficients of the two series.

As with the Cesàro transformation we find a formula for $g$ in terms of $f$, as follows.

$$
\begin{aligned}
g(z) & =\sum_{n=0}^{\infty} b_{n} z^{n}=\sum_{n=0}^{\infty}\left\{\sum_{k=0}^{n}\left(\begin{array}{l}
n \\
k
\end{array}\right) r^{k}(1-r)^{n-k} a_{k}\right\} z^{n} \\
& =\sum_{k=0}^{\infty}\left\{\sum_{n=k}^{\infty}\left(\begin{array}{l}
n \\
k
\end{array}\right) r^{k}(1-r)^{n-k} a_{k} z^{n}\right\}=\sum_{k=0}^{\infty} r^{k} a_{k}\left\{\sum_{n=k}^{\infty}\left(\begin{array}{l}
n \\
k
\end{array}\right)(1-r)^{n-k} z^{n}\right\}
\end{aligned}
$$




$$
=\sum_{k=0}^{\infty} r^{k} a_{k} z^{k}\left\{\sum_{m=0}^{\infty}\left(\begin{array}{c}
m \\
-k-1
\end{array}\right)[-(1-r) z]^{m}\right\}=\sum_{k=0}^{\infty} r^{k} a_{k} z^{k} \frac{1}{[1-(1-r) z]^{k+1}} .
$$

Therefore, we conclude that

$$
g(z)=\frac{1}{1-(1-r) z} f\left(\frac{r z}{1-(1-r) z}\right) .
$$

The interchange in summation used at the beginning of this computation is justified by Weierstrass' double-series theorem.

Let $S$ denote the family of normalized univalent functions; that is, $f \in S$ if $f$ is analytic and univalent in $\Delta$ and if $f(0)=0$ and $f^{\prime}(0)=1$. Since $b_{1}=r a_{1}$ when $f(0)=0$, the Euler-Knopp transform of a function in $S$ satisfies $g^{\prime}(0)=r$ and for convenience, we therefore consider the functions $h(z)=g(z) / r$. The set of all functions $h$ obtained this way by varying $f$ in $S$ shall be denoted $U$. The parameter $r$ is now allowed to varying in the interval $[0,1]$ and we note that if $r=1$ then $h=f$ and if $r=0$ we mean that $h(z)=z /(1-z)^{2}$ since $\lim _{r \rightarrow 0} h(z)=z /(1-z)^{2}$. In our next theorem we show that some of the classical inequalities for functions in $S$ hold for the larger family $U$. The results for $S$ are found in reference [8] and also in [2].

THEOREM 4. If $h \in U$ and $h(z)=z+\sum_{n=2}^{\infty} c_{n} z^{n}$, then

$$
\begin{aligned}
|h(z)| & \leqq \frac{|z|}{(1-|z|)^{2}} \\
\left|h^{\prime}(z)\right| & \leqq \frac{1+|z|}{(1-|z|)^{3}} \\
\left|\frac{z h^{\prime}(z)}{h(z)}\right| & \leqq \frac{1+|z|}{1-|z|} \\
\left|c_{2}^{2}-c_{3}\right| & \leqq 1
\end{aligned}
$$

Furthermore, if $h$ is associated with $f$ and

$$
\begin{aligned}
& f(z)=z+\sum_{n=2}^{\infty} a_{n} z^{n}, \text { then } \\
& \left|a_{k}\right| \leqq k \text { for } k=2,3, \cdots, n
\end{aligned}
$$

implies that

$$
\left|c_{k}\right| \leqq k \text { for } k=2,3, \cdots, n
$$

Proof. Inequality (33) holds for a function $f$ in $S[8$, see page 217$]$, and, therefore, by (32) 


$$
\begin{aligned}
|h(z)| & =\frac{1}{r}|g(z)| \leqq \frac{1}{r} \frac{1}{|1-(1-r) z|} \frac{\left|\frac{r z}{1-(1-r) z}\right|}{\left(1-\left|\frac{r z}{1-(1-r) z}\right|\right)^{2}} \\
& \leqq \frac{1}{r} \frac{1}{1-(1-r)|z|} \frac{\frac{r|z|}{1-(1-r)|z|}}{\left(1-\frac{r|z|}{1-(1-r)|z|}\right)^{2}}=\frac{|z|}{(1-|z|)^{2}} .
\end{aligned}
$$

This proves inequality (33).

To deduce (34) we additionally take advantage of the fact that (34) holds for a function $f$ in $S$ [8; see page 216]. Equation (32) implies that

$$
g^{\prime}(z)=\frac{r}{[1-(1-r) z]^{3}} f^{\prime}\left(\frac{r z}{1-(1-r) z}\right)+\frac{1-r}{[1-(1-r) z]^{2}} f\left(\frac{r z}{1-(1-r) z}\right)
$$

and, therefore,

$$
\begin{aligned}
\left|g^{\prime}(z)\right| \leqq & \frac{r}{|1-(1-r) z|^{3}} \frac{1+\left|\frac{r z}{1-(1-r) z}\right|}{\left(1-\left|\frac{r z}{1-(1-r) z}\right|\right)^{3}} \\
& +\frac{1-r}{|1-(1-r) z|^{2}} \frac{\left|\frac{r z}{1-(1-r) z}\right|}{\left(1-\left|\frac{r z}{1-(1-r) z}\right|\right)^{2}} \\
\leqq & \frac{r}{(1-(1-r)|z|)^{3}} \frac{r|z|}{\left(1-\frac{r|z|}{1-(1-r)|z|}\right)^{3}} \\
& +\frac{1-r}{(1-(1-r)|z|)^{2}} \frac{(1-r)|z|}{\left(1-\frac{r|z|}{1-(1-r)|z|}\right.} \\
= & r \frac{1+|z|}{(1-|z|)^{3}} \cdot
\end{aligned}
$$

Since $h^{\prime}(z)=(1 / r) g^{\prime}(z)$ this proves (34).

Inequality (35) likewise results from the fact it holds for functions in $S[8$; see page 224], as follows. Equation (32) implies that 
and, thus,

$$
\frac{z g^{\prime}(z)}{g(z)}=\frac{r z f^{\prime}\left(\frac{r z}{1-(1-r) z}\right)}{[1-(1-r) z]^{2} f\left(\frac{r z}{1-(1-r) z}\right)}+\frac{(1-r) z}{1-(1-r) z}
$$

$$
\left|\frac{z g^{\prime}(z)}{g(z)}\right| \leqq \frac{1}{|1-(1-r) z|} \cdot \frac{1+\left|\frac{r z}{1-(1-r) z}\right|}{1-\left|\frac{r z}{1-(1-r) z}\right|}+\frac{(1-r)|z|}{|1-(1-r) z|} .
$$

Since the function $y=(x+a) /(x-a)$ decreases for $x>a$ we conclude that

$$
\begin{aligned}
\left|\frac{z g^{\prime}(z)}{g(z)}\right| & \leqq \frac{1}{1-(1-r)|z|} \frac{1-(1-r)|z|+r|z|}{1-(1-r)|z|-r|z|}+\frac{(1-r)|z|}{1-(1-r)|z|} \\
& =\frac{1+(1+r)|z|}{1-(1-r)|z|} .
\end{aligned}
$$

The function of $r$ last written is decreasing on $[0,1]$ and, therefore,

$$
\left|\frac{z g^{\prime}(z)}{g(z)}\right| \leqq \frac{1+|z|}{1-|z|} \text {. }
$$

This proves (35) since

$$
\frac{z h^{\prime}(z)}{h(z)}=\frac{z g^{\prime}(z)}{g(z)} .
$$

In order to prove (36) we take advantage of the inequalities $\left|a_{2}^{2}-a_{3}\right| \leqq 1$ and $\left|a_{2}\right| \leqq 2$, valid for the function $f(z)=z+a_{2} z^{2}+a_{3} z^{3}+\cdots$ belonging to $S$ [8; see page 213]. Namely, equation (8) with $n=2$ and 3 shows that $c_{2}=2(1-r)+r a_{2}$ and $c_{3}=3(1-r)^{2}+3 r(1-r) a_{2}+r^{2} a_{3}$ and hence

$$
c_{2}^{2}-c_{3}=(1-r)^{2}+r(1-r) a_{2}+r^{2}\left(a_{2}^{2}-a_{3}\right) \text {. }
$$

Therefore, $\left|c_{2}^{2}-c_{3}\right| \leqq(1-r)^{2}+2 r(1-r)+r^{2}=1$.

Inequality (38) also follows from (8), given (37), as follows. If $k \leqq n$ then

$$
\left|b_{k}\right| \leqq \sum_{j=1}^{k}\left(\begin{array}{l}
k \\
j
\end{array}\right) r^{j}(1-r)^{n-j} j=k r
$$

The last equality is a consequence of differentiating the identity at $x=1$ :

$$
[(1-r)+r x]^{k}=\sum_{j=0}^{k}\left(\begin{array}{l}
k \\
j
\end{array}\right)(r x)^{j}(1-r)^{n-j} .
$$

This proves (38) as $c_{k}=(1 / r) b_{k}$. 
We add the remark that if (37) holds for all $k$ then so does (38). This is the case, for example, if $f$ is close-to-convex [9] or if $f$ has real coefficiencts $a_{k}[8$, page 220]. Also, since the Bieberbach conjecture (that is, equation (37)) holds for $k=2,3,4,5$ and 6 so does equation (38). Also, any estimate of the form $\left|a_{n}\right| \leqq A \cdot n(n=2,3, \cdots)$ for the coefficients of a function in $S$ implies the same estimate $\left|c_{n}\right| \leqq A \cdot n$ on the coefficients of functions in $U$. For example, Littlewood's classical result $\left|a_{n}\right|<e n\left[8\right.$, page 218] implies that $\left|c_{n}\right|<e n$.

We now give a general condition under which the Euler-Knopp transformation of a univalent function is univalent, namely, when $f$ is a starlike mapping. Our proof depends upon Lemma 1 and the following result. This result is generally known and is a consequence of a similar assertion for functions with a positive real part in $\Delta$ as given by the Herglotz formula. We introduce the notation $S t$ to denote the collection of all starlike mappings in $S$. Thus, $f \in S t$ if $f$ is analytic and univalent in $\Delta, f(0)=0, f^{\prime}(0)=1$ and $f(\Delta)$ is starlike with respect to the origin.

LEMMA 2. If $f \in S t$ and $\rho$ is given $(0<\rho<1)$, then $f$ can be uniformly approximated in $|z| \leqq \rho$ by functions of the form

and

$$
F(z)=\frac{z}{\prod_{k=1}^{n}\left(1-\varepsilon_{k} z\right)^{\lambda_{k}}} \text {, where }\left|\varepsilon_{k}\right|=1, \lambda_{k} \geqq 0
$$

$$
\sum_{k=1}^{n} \lambda_{k}=2
$$

THEOREM 5. If $f \in S t$ and $g$ is the Euler-Knopp transformation of $f$, then $h=g / r \in S t$.

ProOF. If $f \in S t$ then $f$ can be approximated by functions $F$ given by Lemma 2 . The Euler-Knopp transformation $g$ of $f$ is thereby approximated by the functions $G$ that are the Euler-Knopp transformations of the functions $F$. If we show that each such $G$ is starlike, then $\operatorname{Re} z G^{\prime}(z) / G(z)>0$ in $\Delta$ and, as a consequence, $\operatorname{Re} z g^{\prime}(z) / g(z) \geqq 0$ or, equivalently, $\operatorname{Re} z h^{\prime}(z) / h(z) \geqq 0$. This implies that $\operatorname{Re} z h^{\prime}(z) / h(z)>0$ in $\Delta$ as the equality $\operatorname{Re} z h^{\prime}(z) / h(z)=0$ at some $z$ in $\Delta$ implies, by the minimum principle for harmonic functions, that $\operatorname{Re} z h^{\prime}(z) / h(z)=0$ at all $z$ in $\Delta$ contrary to the fact that $\operatorname{Re} z h^{\prime}(z) / h(z)=1$ at $z=0$. This shows that we need only show that $\operatorname{Re} z g^{\prime}(z) / g(z)>0$ in $\Delta$ where $g$ is the Euler-Knopp transformation of the function

$$
f(z)=\frac{z}{\prod_{k=1}^{n}\left(1-\varepsilon_{k} z\right)^{\lambda_{k}}}, \text { where }\left|\varepsilon_{k}\right|=1, \lambda_{k} \geqq 0 \text { and } \sum_{k=1}^{n} \lambda_{k}=2 .
$$

When $f$ has this form equation (32) implies that 


$$
g(z)=\frac{r z}{\prod_{k=1}^{n}\left(1-\delta_{k} z\right)^{\lambda_{k}}}
$$

where $\delta_{k}=1-r+\varepsilon_{k} r$. Since $\left|\delta_{k}\right| \leqq 1$ we see that $h=g / r$ has the form of Lemma 1 and thus $h \in S t$.

Although the Euler-Knopp transformation maps a starlike, univalent function onto another one it does not preserve convex mappings. This can be seen in various ways. For example, since the normalized Euler-Knopp transformation $h(z) \rightarrow k(z)$ $=z /(1-z)^{2}$ as $r \rightarrow 0$ we find that the expression $z h^{\prime \prime}(z) / h^{\prime}(z)+1$ is approximated by $z k^{\prime \prime}(z) / k^{\prime}(z)+1$ uniformly in $|z| \leqq \rho$ as $r \rightarrow 0$. Now, the condition

$$
\operatorname{Re}\left\{\frac{z h^{\prime \prime}(z)}{h^{\prime}(z)}+1\right\}>0, \quad|z|<\rho
$$

is necessary and sufficient for $h$ to be univalent and convex in $|z|<\rho$ (given that $\left.h^{\prime}(0) \neq 0\right)$. Thus, it is clear that if $k$ is not univalent and convex in $|z|<\rho$ neither is $h$ for $r$ sufficiently close to 0 . The radius of convexity of $k$ is $2-\sqrt{ } 3$ and, thus, independent of $r$, we can at best assert that each function $h$ is univalent and convex for $|z|<2-\sqrt{3}$ (independent of whether $f$ is convex in $\Delta$ ).

We next present a proof that the Euler-Knopp transformation of a function in $S$ is not always univalent. It depends upon the following assertions.

LEMma 3. The set $\left\{w: w=z f^{\prime}(z) / f(z),|z|<1, f \in S\right\}$ consists of all complex numbers except $w=0$.

LEMma 4. The set $\left\{w: w=x f^{\prime}(x) / f(x), 0 \leqq x<1, f \in S\right\}$ consists of all complex numbers except $w=0$.

These results are quite expected and probably are easy consequences of the more delicate information regarding the exact region of variability of $z f^{\prime}(z) / f(z)$ with $z$ fixed and $f$ varying over $S$. Our argument is quite direct and affords an explicit construction of a function $f$ in $S$ so that $h \notin S$.

To prove Lemma 3 we consider the functions (19) where (20) holds so that $f \in S$. From (19) we find that

$$
\frac{z f^{\prime}(z)}{f(z)}=\frac{1+(1-2 a) z}{(1-a z)(1-z)} .
$$

We further restrict our consideration so that $\left|a-\frac{1}{2}\right|=\frac{1}{2}$, and letting $a=\frac{1}{2}$ $+\frac{1}{2} e^{i \phi}$ and $z=\rho e^{i \theta}(0 \leqq \rho<1)$ we may write (40) into the form

$$
w=\frac{z f^{\prime}(z)}{f(z)}=\frac{2 v}{(u+v) u}
$$

where $u=1-\rho e^{i \theta}$ and $v=1-\rho e^{i(\theta+\phi)}$. If we let $\zeta=1 / w$ then (41) implies that 


$$
\zeta=\frac{1}{2} u\left(1+\frac{u}{v}\right)
$$

By varying $\phi, \theta$ and $\rho$ we find that $u$ and $v$ independently vary over the disk $D=\{z:|z-1|<1\}$. Thus, the numbers $1 / v$ vary over the half-plane $\operatorname{Re} z>\frac{1}{2}$. If each point $1 / v$ in this half-plane is multiplied by the points $u$ in $D$ with the same argument $\beta$ a half-plane results perpendicular to the direction $\beta$. A similar halfplane results if this half-plane is translated by 1 . Because of the factor $u$ in (42) we conclude that if $u$ varies in $D$ with a direction $\beta$ and $v$ freely varies in $D$, then the numbers $\zeta$ form an open half-plane through $\zeta=0$ perpendicular to the direction $2 \beta$. Since we can then vary $\beta$ in the interval $|\beta|<\pi / 2$ we find that the union of these half-planes covers the whole complex plane except $\zeta=0$. The same can be said of the numbers $w=1 / \zeta$ and this proves Lemma 3 .

Lemma 4 is a consequence of Lemma 3 and the fact that if $f \in S$ and $g(z)$ $=(1 / \varepsilon) f(\varepsilon z)$ then $g \in S$ for each complex number $\varepsilon,|\varepsilon|=1$. The relation between $f$ and $g$ is equivalent to $f(z)=(1 / \delta) g(\delta z)$ where $\delta=1 / \varepsilon$ and so $z f^{\prime}(z) / f(z)$ $=\delta z g^{\prime}(\delta z) / g(\delta z)$. Thus, any number $z f^{\prime}(z) / f(z)$ where $f \in S$ and $z \in \Delta$ is also obtained in the form $x g^{\prime}(x) / g(x)$ where $g \in S$ and $0 \leqq x<1$ simply by choosing $\delta$ so that $\delta z=|z|=x$.

THEOREM 6. There is a function $f$ in $S$ so that the Euler-Knopp transformation $g$ of $f$ is not univalent in $\Delta$ for some values of $r$. In other words, $U \notin S$.

Proof. Since the derivative of a univalent function does not vanish, we obtain our conclusion by showing that there is a function $f$ in $S$ and a number $r$ $(0<r<1)$ so that $g^{\prime}(z)=0$ at some $z(|z|<1)$. Because of equation (32) the condition $g^{\prime}(z)=0$ is equivalent to

$$
\frac{f^{\prime}\left(\frac{r z}{1-(1-r) z}\right)}{f\left(\frac{r z}{1-(1-r) z}\right)}=-\frac{1-r}{r}[1-(1-r) z] .
$$

If we let $w=r z /[1-(1-r) z]$, then (43) may be written in the form

$$
\frac{w f^{\prime}(w)}{f(w)}=-\frac{(1-r) w}{r+(1-r) w} .
$$

If $y$ is any real number and $-1<y<0$ then by Lemma 4 there is a function $f$ in $S$ and a real number $x$ so that $0<x<1$ and $x f^{\prime}(x) / f(x)=y$. The function

$$
u(r)=-\frac{(1-r) x}{r+(1-r) x}
$$

is continuous in $r$ on $[0,1]$, given $x$ so that $0<x<1$. Thus, as $u(0)=-1$ and $u(1)=0$ there is a number $r_{0}$ so that $u\left(r_{0}\right)=y$ and $0<r_{0}<1$. With this choice of 
$f$ and with $r=r_{0}$ we see that equation (44) holds with $w=x$. Therefore, the Euler-Knopp transformation of $f$ is not univalent in $\Delta$ if $r=r_{0}$.

REMARKS. 1. Our proof of Theorem 6 is quite constructive because of the explicit functions used to prove Lemma 3. We thereby can exhibit functions $f(z)=\left(z-\varepsilon a z^{2}\right) /(1-\varepsilon z)^{2}$ where $\left|a-\frac{1}{2}\right|=\frac{1}{2}$ and $|\varepsilon|=1$ which have EulerKnopp transformations which are not univalent in $\Delta$ for various values of $r$.

2. If one relaxes the condition that $f \in S$ to $f$ is univalent and analytic in $\Delta$, then there is a simple example of the fact that there is an $f$ univalent in $\Delta$ whose Euler-Knopp transform $g$ is not univalent. Let $f(z)=r /(r+[1-r] z), 0<r<1$. Then $f$ is univalent in $\Delta$ but $f \notin S$ and

$$
g(z)=\frac{1}{1-(1-r) z} \cdot f\left(\frac{r z}{1-(1-r) z}\right) \equiv 1,
$$

which is clearly not univalent.

3. We have chosen to discuss the two specific matrices given by (6) and (7) because they exhibit the interesting results concerning univalence that we have presented here. There are other matrices which may also be worth considering in this way.

For example, the Cesàro matrix transforms $f$ into $g$ so that (11) holds. This may be expressed in the form

$$
\frac{z g^{\prime}(z)}{f(z)}=p(z)
$$

where $p(z)=1 /(1-z)$. As the proof of Theorem 2 shows, if simply $\operatorname{Re} p(z)>0$ then $g$ is univalent in $\Delta$ for each function $f$ univalent and starlike in $\Delta$ (and $f(0)$ $=0$ ). Thereby, each function $p$ with a positive real part produces a transformation from $f$ to $g$ which already maps certain univalent functions into univalent functions. Such a function $p$ is uniquely given by a matrix expressed in terms of the coefficients of the power series for $p$. Examples of such "generalized Cesàro matrices" are obtained from the functions $w=(1+z) /(1-z), w=[(1+z) /(1-z)]^{\alpha}$ $(0 \leqq \alpha \leqq 1)$,

$$
w=\frac{1}{1-z^{n}}, \text { or } w=\left(\frac{1}{1-z}\right)^{\alpha}(0<\alpha \leqq 1) .
$$

4. We indicate here what happens if the Euler-Knopp matrix is "shifted." Namely, suppose that $0<r<1$ and define the matrix $A=\left(\alpha_{n k}\right)$ by

$$
\alpha_{n k}= \begin{cases}\left(\begin{array}{l}
n-1 \\
k-1
\end{array}\right) r^{k}(1-r)^{n-k}, & \text { if } k \leqq n \\
0, & \text { if } k>n .\end{cases}
$$

This transforms the power series (4) into (5) where 


$$
b_{n}=\sum_{k=1}^{n}\left(\begin{array}{l}
n-1 \\
k-1
\end{array}\right) r^{k}(1-r)^{n-k} a_{k} \text {. }
$$

From this we can show that

$$
g(z)=f\left(\frac{r z}{1-(1-r) z}\right)
$$

proceeding as in the derivation of equation (33). Since $0<r<1$ and $|z|<1$ imply that $w=r z /(1-(1-r) z)$ belongs to $\Delta$, it is then clear that if $f$ is univalent in $\Delta$ then so is its ("shifted") Euler-Knopp transformation $g$.

\section{The Taylor Transformation}

THEOREM 7. If $f(z)=\sum_{k=0}^{\infty} a_{k} z^{k}$ is analytic and univalent in $\Delta$ then $g(z)$ $=\sum_{n=0}^{\infty} b_{n} z^{n}$, the Taylor transformation of $f$ where the $b_{n}$ 's are given by (9), is univalent in $\Delta$.

$$
\text { Proof. } \quad \begin{aligned}
g(z) & =\sum_{n=0}^{\infty} b_{n} z^{n}=\sum_{n=0}^{\infty}\left(\sum_{k=n}^{\infty}(1-r)^{n+1}\left(\begin{array}{l}
k \\
n
\end{array}\right) r^{k-n} a_{k}\right) z^{n} \\
& =(1-r) \cdot \sum_{k=1}^{\infty} a_{k}\left(\sum_{n=0}^{\infty}\left(\begin{array}{l}
k \\
n
\end{array}\right)(1-r)^{n} r^{k-n} z^{n}\right) \\
& =(1-r) \sum_{k=1}^{\infty} a_{k}[r+(1-r) z]^{k} \\
& =(1-r) f(r+(1-r) z) .
\end{aligned}
$$

The interchange in summation of the series involved is permissible since for $|z|<1,|r+(1-r) z|<1(0<r<1)$. Hence, the hypothesis on $f$ implies the result.

One should note that $f \in S$ does not imply $g \in S$, since $g$ is not properly normalized. However, one can obtain the various coefficient inequalities of section 2 for $g$, by using the techniques of Theorem 4 .

\section{References}

[1] V. F. Cowling, 'Summability and Analytic Continuation', Proc. Amer. Math. Soc. 1 (1950), 536-542.

[2] W. K. Hayman, Multivalent Functions, (Cambridge University Press, Cambridge, 1958).

[3] W. Kaplan, 'Close to convex schlicht functions', Michigan Math. J. 1(1952), 169-185.

[4] J. Krzyz and Z. Lewandowski, 'On the integral of univalent functions', Bull. Acad. Polon. Sci. Sér. Sci. Math. Astronom. Phys. 11 (1963), 447-448.

[5] T. H. MacGregor, 'Certain integrals of univalent and convex functions', Math. Zeitschr. 103 (1968), 48-54. 
[6] I. J. Maddox, Elements of Funcrional Analysis, (Cambridge University Press, Cambridge, 1970.)

[7] A. Marx, 'Untersuchungen über schlichte Abbildungen', Math. Ann. 107 (1932), $40-67$.

[8] Z. Nehari, Conformal Mapping, (McGraw-Hill, New York, 1952).

[9] M. O. Reade, 'On close-to-convex univalent functions', Michigan Math. J. 3(1955), 59-62.

[10] L. Spacek, 'Contribution à la théorie des fonctions univalentes', Casopis Pest. Mat. 62 (1932), 12-19.

[11] E. Strohhäcker, 'Beitrage zur Theorie der schlichten Funktionen', Math. Zeitschr. 37(1933), 356-380.

[12] T. Umezawa, 'On the theory of univalent functions', Tohoku Math. J. 7 (1955), 212-228.

Villanova University

U. S. A.

and

State University of New York at Albany

U. S. A. 